九州大学学術情報リポジトリ

Kyushu University Institutional Repository

\title{
AN ALGORITHM FOR DETERMINING A CLASS OF TW0- PERSON GAMES HAVING A PURE-STRATEGY NASH EQUILIBRIUM
}

Sato, Jun-ichi

Collaborative Research Team for Verification, National Institute of Advanced Industrial

Science and Technology (AIST)

Ito, Takahiro

Graduate School of Mathematics, Kyushu University

https://doi.org/10.5109/21046

出版情報: Bulletin of informatics and cybernetics. 41, pp.51-58, 2009-12. Research Association of Statistical Sciences

バージョン :

権利関係 : 
AN ALGORITHM FOR DETERMINING A CLASS OF TWO-PERSON GAMES HAVING A PURE-STRATEGY NASH EQUILIBRIUM

by

Jun-ichi SAто and Takahiro Iто

Reprinted from the Bulletin of Informatics and Cybernetics Research Association of Statistical Sciences, Vol.41

FUKUOKA, JAPAN

2009 


\title{
AN ALGORITHM FOR DETERMINING A CLASS OF TWO-PERSON GAMES HAVING A PURE-STRATEGY NASH EQUILIBRIUM
}

\author{
By \\ Jun-ichi SAто* and Takahiro Iто ${ }^{\dagger}$
}

\begin{abstract}
Sato and Kawasaki (Preprint) introduced a class of $n$-person games called partially monotone games, and showed that any partially monotone game has a purestrategy Nash equilibrium. Further, they proved that partial monotonicity is necessary for the existence of a pure-strategy Nash equilibrium in the case of two persons. In this paper, we present an algorithm for determining whether a twoperson game belongs to the class. Our algorithm requires $O\left(m^{2} n^{2}\right)$ time, where $m$ and $n$ are the number of pure strategies of players 1 and 2, respectively.
\end{abstract}

Key Words and Phrases: pure strategy, Nash equilibrium, monotonicity of the best responses

\section{Introduction}

A Nash equilibrium is one of the most important solution concepts in non-cooperative games. Nash (1950) and Nash (1951) showed that if each player uses a mixedstrategy, any non-cooperative game has a Nash equilibrium. A pure-strategy Nash equilibrium, on the other hand, does not always exist. Therefore, some authors dealt with sufficient conditions for the existence of a pure-strategy Nash equilibrium. The first result is due to Topkis (1979). He introduced the so-called supermodular games. He first got monotonicity of the greatest and least element of each player's best response by assuming the property of increasing differences for each player's payoff function. Next, relying on Tarski's fixed point theorem, he showed the existence in supermodular games. Sato and Kawasaki (2009) introduced the so-called monotone game. They provided a discrete fixed point theorem based on monotonicity of a set-valued mapping, and as its application, showed that any monotone game has a pure-strategy Nash equilibrium. The common idea of Sato and Kawasaki (2009) and Topkis (1979) is monotonicity of the best responses.

In Sato and Kawasaki (Preprint), they introduced the so-called partially monotone game (see Definition 2.1), and showed this game has a pure-strategy Nash equilibrium (see Theorem 2.2), which is an extension of the result of Sato and Kawasaki (2009). They also showed that partial monotonicity of the best responses is necessary for the

\footnotetext{
* Collaborative Research Team for Verification, National Institute of Advanced Industrial Science and Technology (AIST); 5th Floor, Mitsui Sumitomo Kaijo Senri Bldg., 1-2-14 Shin-Senri Nishi, Toyonaka, Osaka 560-0083, JAPAN. E-mail: jun-ichi@ni.aist.go.jp

$\dagger$ Graduate School of Mathematics, Kyushu University; 744, Motooka, Nishi-ku, Fukuoka 819-0395, JAPAN. E-mail: t-ito@math.kyushu-u.ac.jp
} 
existence in the case of two persons. Thus, the class of partially monotone games can be regarded as a wide range of two-person games having a pure-strategy Nash equilibrium.

However, it is not efficient to check whether a game belongs to this class directly from its definition. Hence the aim of this paper is presenting an efficient algorithm for determining whether a two-person game is a partially monotone game.

In this paper, we consider the following two-person game $G=\left\{\left\{S_{1}, S_{2}\right\},\{A, B\}\right\}$ :

- $S_{1}:=\{1, \ldots, m\}$ is the set of pure strategies of player 1 , where $m \in \mathbb{N}$.

- $S_{2}:=\{1, \ldots, n\}$ is the set of pure strategies of player 2 , where $n \in \mathbb{N}$.

- $A=\left(a_{i j}\right)$ is a payoff matrix of player 1 .

- $B=\left(b_{i j}\right)$ is a payoff matrix of player 2 .

If we impromptu check whether a two-person game is a partially monotone game, we need $O\left(2^{m+n} m ! n !\right)$ time in general. In Section 3, we prove any partially monotone game contains a small-size subgame that is also a partially monotone game. Then our algorithm requires only $O\left(m^{2} n^{2}\right)$ time in total.

This paper consists of five sections. In Section 2, we recall the class of partially monotone games and the existence theorem of a pure-strategy Nash equilibrium given by Sato and Kawasaki (Preprint). Section 3 provides a useful property of the class for computing. Section 4 develops an algorithm for determining whether a two-person game belongs to the class. Finally, Section 5 is concluding remarks.

Notation Throughout this paper, let $F_{1}(j)$ and $F_{2}(i)$ be the set of best responses of players 1 and 2, respectively, that is,

$$
\begin{aligned}
& F_{1}(j):=\left\{i^{\prime} \in S_{1}: a_{i^{\prime} j}=\max _{i \in S_{1}} a_{i j}\right\} \text { for any } j \in S_{2}, \\
& F_{2}(i):=\left\{j^{\prime} \in S_{2}: b_{i j^{\prime}}=\max _{j \in S_{2}} b_{i j}\right\} \text { for any } i \in S_{1} .
\end{aligned}
$$

Further, $F(i, j):=F_{1}(j) \times F_{2}(i)$ denotes the set of best responses of $(i, j) \in S_{1} \times S_{2}$. Then a pair $\left(i^{*}, j^{*}\right)$ is a pure-strategy Nash equilibrium if and only if $\left(i^{*}, j^{*}\right) \in F\left(i^{*}, j^{*}\right)$.

\section{Preliminaries}

We first recall the definition of a partially monotone game from Sato and Kawasaki (Preprint). Although Sato and Kawasaki (Preprint) dealt with $n$-person games, we consider only two-person games in this paper.

Let $T_{k}$ be a non-empty subset of $S_{k}$. For any permutation $\sigma_{k}$ on $T_{k}$, we define a total order $s_{k} \leqq \sigma_{k} t_{k}$ on $T_{k}$ by $\sigma_{k}\left(s_{k}\right) \leqq \sigma_{k}\left(t_{k}\right)$. Further, $s_{k}<_{\sigma_{k}} t_{k}$ means $\sigma_{k}\left(s_{k}\right)<\sigma_{k}\left(t_{k}\right)$. For any $\sigma=\left(\sigma_{1}, \sigma_{2}\right), s=\left(s_{1}, s_{2}\right)$ and $t=\left(t_{1}, t_{2}\right), s \varliminf_{\sigma} t$ means $s_{k} \leqq_{\sigma_{k}} t_{k}$ for all $k=1,2$. The symbol $s \preceq_{\sigma} t$ means $s \varliminf_{\sigma} t$ and $s \neq t$.

DeFinition 2.1. We call $G$ a partially monotone game if there exist a selection $f$ of $F$, non-empty subsets $T_{k} \subset S_{k}$ and permutations $\sigma_{k}$ on $T_{k}(k=1,2)$ such that at least one of $T_{k}$ 's has two or more elements, $f\left(T_{1} \times T_{2}\right) \subset T_{1} \times T_{2}$,

$$
j^{0}<_{\sigma_{2}} j^{1} \Rightarrow f_{1}\left(j^{0}\right) \leqq \sigma_{1} f_{1}\left(j^{1}\right) \text { for any } j^{0}, j^{1} \in T_{2}
$$


and

$$
i^{0}<_{\sigma_{1}} i^{1} \Rightarrow f_{2}\left(i^{0}\right) \leqq \sigma_{2} f_{2}\left(i^{1}\right) \text { for any } i^{0}, i^{1} \in T_{1} .
$$

For the existence of a pure-strategy Nash equilibrium, we recall the following:

TheOREM 2.2. Any partially monotone game has a pure-strategy Nash equilibrium in $T:=T_{1} \times T_{2}$.

\section{A key lemma on partially monotone games}

We first define the term of "isolated Nash equilibrium."

Definition 3.1. A Nash equilibrium $\left(i^{*}, j^{*}\right)$ is said to be isolated if $\left(i^{*}, j^{*}\right) \in$ $F(i, j)$ implies $(i, j)=\left(i^{*}, j^{*}\right)$.

Since $F$ has a separated form $F_{1} \times F_{2}$, isolated Nash equilibria are characterized as the lemma below. Further, the following implies that when $\left(i^{*}, j^{*}\right)$ is an isolated Nash equilibrium, the best response operation is closed in $\left(S_{1} \backslash\left\{i^{*}\right\}\right) \times\left(S_{2} \backslash\left\{j^{*}\right\}\right)$.

Lemma 3.2. A Nash equilibrium $\left(i^{*}, j^{*}\right)$ is isolated if and only if both $i^{*} \notin F_{1}(j)$ for any $j \neq j^{*}$, and $j^{*} \notin F_{2}(i)$ for any $i \neq i^{*}$ hold. Then it holds that

$$
F\left(\left(S_{1} \backslash\left\{i^{*}\right\}\right) \times\left(S_{2} \backslash\left\{j^{*}\right\}\right)\right) \subset\left(S_{1} \backslash\left\{i^{*}\right\}\right) \times\left(S_{2} \backslash\left\{j^{*}\right\}\right) .
$$
$F_{2}\left(i^{*}\right)$.

Proof. Since $\left(i^{*}, j^{*}\right)$ is a Nash equilibrium, it holds that $i^{*} \in F_{1}\left(j^{*}\right)$ and $j^{*} \in$

("only if" part) Suppose that $i^{*} \in F_{1}(j)$ for some $j \neq j^{*}$. Then $\left(i^{*}, j^{*}\right) \in F_{1}(j) \times$ $F_{2}\left(i^{*}\right)$ and $\left(i^{*}, j\right) \neq\left(i^{*}, j^{*}\right)$, which contradicts the assumption. Hence $i^{*} \notin F_{1}(j)$ for any $j \neq j^{*}$. Similarly, $j^{*} \notin F_{2}(i)$ for any $i \neq i^{*}$. ("if" part) If $\left(i^{*}, j^{*}\right) \in F_{1}(j) \times F_{2}(i)$, then, by the assumption, we have $j=j^{*}$ and $i=i^{*}$, that is, $\left(i^{*}, j^{*}\right)$ is isolated. The second claim is evident from the first claim.

The next lemma will be a key in the proof of Theorem 3.5 below.

Lemma 3.3. Let $G$ be a partially monotone game, and $T_{k}(k=1,2)$ be corresponding subsets of pure strategies mentioned in Definition 2.1. If $G$ has an isolated pure-strategy Nash equilibrium in $T=T_{1} \times T_{2}$, then it has another pure-strategy Nash equilibrium in $T$, which neither first nor second element coincides with that of the isolated equilibrium.

Proof. Let $\left(i^{*}, j^{*}\right)$ be an isolated pure-strategy Nash equilibrium in $T$. Then it follows from Lemma 3.2 that $F\left(T^{\prime}\right) \subset T^{\prime}$, where $T_{1}^{\prime}:=T_{1} \backslash\left\{i^{*}\right\}, T_{2}^{\prime}:=T_{2} \backslash\left\{j^{*}\right\}$ and $T^{\prime}:=T_{1}^{\prime} \times T_{2}^{\prime}$. Hence $\# T_{1} \geq 2$ and $\# T_{2} \geq 2$.

We first consider the case where $\# T_{1}=\# T_{2}=2$, then $T^{\prime}$ is a single point set. Since $F\left(T^{\prime}\right) \subset T^{\prime}$, the point is the pure-strategy Nash equilibrium we want.

Next, we consider the case where $\# T_{k} \geq 3$ for some $k \in\{1,2\}$. By the definition of a partially monotone game, there exist a selection $f$ of $F$ and permutations $\sigma_{k}$ on $T_{k}(k=1,2)$ such that $f(T) \subset T$,

$$
j^{0}<_{\sigma_{2}} j^{1} \Rightarrow f_{1}\left(j^{0}\right) \leqq \sigma_{1} f_{1}\left(j^{1}\right) \text { for any } j^{0}, j^{1} \in T_{2},
$$


and

$$
i^{0}<_{\sigma_{1}} i^{1} \Rightarrow f_{2}\left(i^{0}\right) \leqq \sigma_{2} f_{2}\left(i^{1}\right) \text { for any } i^{0}, i^{1} \in T_{1} .
$$

Align the elements of $T_{1}$ as $i_{1}<i_{2}<\cdots<i_{m_{1}}$. Then, since $i^{*}$ and $\sigma\left(i^{*}\right)$ belong to $T_{1}$, they can be expressed as $i^{*}=i_{p}$ and $\sigma\left(i^{*}\right)=i_{q}$ for some $1 \leq p, q \leq m_{1}$, respectively. Here we define a permutation $\hat{\sigma}_{1}$ on $T_{1}^{\prime}$ as follows.

Case 1: When $p=q$, we define $\hat{\sigma}_{1}(i):=\sigma_{1}(i)$ for any $i \in T_{1}^{\prime}$.

Case 2: When $p>q$, we define

$$
\hat{\sigma}_{1}\left(\sigma_{1}^{-1}\left(i_{m}\right)\right):= \begin{cases}i_{m-1}, & \text { if } q+1 \leq m \leq p \\ i_{m}, & \text { otherwise }\end{cases}
$$

Note that the meaning of $\hat{\sigma}_{1}$ is illustrated in Example 3.4 below.

Case 3: When $p<q$, we define

$$
\hat{\sigma}_{1}\left(\sigma_{1}^{-1}\left(i_{m}\right)\right):= \begin{cases}i_{m+1}, & \text { if } p \leq m \leq q-1 \\ i_{m}, & \text { otherwise. }\end{cases}
$$

In the same way, we define a permutation $\hat{\sigma}_{2}$ on $T_{2}^{\prime}$, and by (1) and (2), it follows that

$$
\begin{aligned}
& j^{0}<\hat{\sigma}_{2} j^{1} \Rightarrow f_{1}\left(j^{0}\right) \leqq \hat{\sigma}_{1} f_{1}\left(j^{1}\right) \text { for any } j^{0}, j^{1} \in T_{2}^{\prime}, \\
& i^{0}<_{\hat{\sigma}_{1}} i^{1} \Rightarrow f_{2}\left(i^{0}\right) \leqq \hat{\sigma}_{2} f_{2}\left(i^{1}\right) \text { for any } i^{0}, i^{1} \in T_{1}^{\prime} .
\end{aligned}
$$

Then the subgame $G^{\prime}=\left\{\left\{T_{1}^{\prime}, T_{2}^{\prime}\right\},\left\{A^{\prime}, B^{\prime}\right\}\right\}$ is a partially monotone game. Therefore, by Theorem 2.2, there exists a pure-strategy Nash equilibrium, say, $\left(i^{\prime}, j^{\prime}\right)$ in $T^{\prime}$. Since $i^{*} \notin T_{1}^{\prime}$ and $j^{*} \notin T_{2}^{\prime},\left(i^{\prime}, j^{\prime}\right)$ is a pure-strategy Nash equilibrium we want.

Example 3.4. Let $T_{1}=\{2,4,6,8,10,12,14\}, i^{*}=12$ and

$$
\sigma_{1}=\left(\begin{array}{ccccccc}
2 & 4 & 6 & 8 & 10 & 12 & 14 \\
14 & 2 & 10 & 12 & 8 & 4 & 6
\end{array}\right)=\left(\begin{array}{ccccccc}
4 & 12 & 14 & 10 & 6 & 8 & 2 \\
2 & 4 & 6 & 8 & 10 & 12 & 14
\end{array}\right) .
$$

Then $T_{1}^{\prime}:=\{2,4,6,8,10,14\}, p=6>2=q, i_{p}=12$ and $i_{q}=\sigma_{1}\left(i_{p}\right)=4$. This is Case 2 in the proof of Lemma 3.3, and our goal is deleting number 12 from the rage of $\sigma_{1}$ by renumbering. First, since there is no $i \in T_{1}^{\prime}$ such that $\sigma_{1}(i)=4$, we define $\hat{\sigma}_{1}\left(\sigma_{1}^{-1}(6)\right)=\hat{\sigma}_{1}(14):=4$. By this procedure, since there does not exist $i$ such that $\sigma_{1}(i)=6$, we define $\hat{\sigma}_{1}\left(\sigma_{1}^{-1}(8)\right)=\hat{\sigma}(10):=6$. Repeating this procedure until we meet $i$ such that $\sigma_{1}(i)=12$, we can delete number 12 from the range of $\sigma_{1}$. We define $\hat{\sigma}_{1}=\sigma_{1}$ for numbers not appearing the procedure above. Then $\hat{\sigma}$ is as follows:

$$
\hat{\sigma}_{1}=\left(\begin{array}{cccccc}
2 & 4 & 6 & 8 & 10 & 14 \\
14 & 2 & 8 & 10 & 6 & 4
\end{array}\right) .
$$

Any partially monotone game has the property that it contains at least one $1 \times 2$, $2 \times 1$ - or $2 \times 2$-subgame that is also a partially monotone game. This is a key property for developing our algorithm in the next section.

TheOrem 3.5. A game $G$ is a partially monotone game if and only if there exist a selection $f$ of $F, R_{1} \subset S_{1}$ and $R_{2} \subset S_{2}$ such that one of the following holds: 
An algorithm for determining a class of two-person games having a pure-strategy Nash equilibrium 55

(i) $\# R_{1}=1$, \# $R_{2}=2$ and $f\left(R_{1} \times R_{2}\right) \subset R_{1} \times R_{2}$;

(ii) $\# R_{1}=2, \# R_{2}=1$ and $f\left(R_{1} \times R_{2}\right) \subset R_{1} \times R_{2}$;

(iii) there exists a permutation $\sigma_{2}$ on $R_{2}$ such that $\# R_{1}=\# R_{2}=2, f\left(R_{1} \times R_{2}\right) \subset$ $R_{1} \times R_{2}$

$$
j<_{\sigma_{2}} j^{\prime} \Rightarrow f_{1}(j) \varliminf_{\text {id }} f_{1}\left(j^{\prime}\right) \text { for any } j, j^{\prime} \in R_{2},
$$

and

$$
i<_{i d} i^{\prime} \Rightarrow f_{2}(i) \leqq_{\sigma_{2}} f_{2}\left(i^{\prime}\right) \text { for any } i, i^{\prime} \in R_{1}
$$

Proof. Since "if" part is evident from the definition of a partially monotone game, we only prove "only if" part. Suppose that $G=\left\{\{A, B\},\left\{S_{1}, S_{2}\right\}\right\}$ is a partially monotone game. Then, by Theorem 2.2, there exists a pure-strategy Nash equilibrium in $T$, say, $\left(i^{*}, j^{*}\right)$. Define

$$
f_{1}\left(j^{*}\right)=i^{*}, f_{2}\left(i^{*}\right)=j^{*}
$$

Case 1: When $\left(i^{*}, j^{*}\right)$ is isolated, by Lemma 3.3, there exists another pure-strategy Nash equilibrium $\left(i^{\star}, j^{\star}\right)$ in $T$ such that $i^{*} \neq i^{\star}$ and $j^{*} \neq j^{\star}$. Taking $R_{1}=\left\{i^{*}, i^{\star}\right\}$, $R_{2}=\left\{j^{*}, j^{\star}\right\}, f_{1}\left(j^{\star}\right)=i^{\star}$ and $f_{2}\left(i^{\star}\right)=j^{\star}$, we have

$$
\begin{aligned}
& f\left(i^{*}, j^{*}\right):=\left(f_{1}\left(j^{*}\right), f_{2}\left(i^{*}\right)\right)=\left(i^{*}, j^{*}\right) \in F\left(i^{*}, j^{*}\right), \\
& f\left(i^{*}, j^{\star}\right):=\left(f_{1}\left(j^{\star}\right), f_{2}\left(i^{*}\right)\right)=\left(i^{\star}, j^{*}\right) \in F\left(i^{*}, j^{\star}\right), \\
& f\left(i^{\star}, j^{*}\right):=\left(f_{1}\left(j^{*}\right), f_{2}\left(i^{\star}\right)\right)=\left(i^{*}, j^{\star}\right) \in F\left(i^{\star}, j^{*}\right), \\
& f\left(i^{\star}, j^{\star}\right):=\left(f_{1}\left(j^{\star}\right), f_{2}\left(i^{\star}\right)\right)=\left(i^{\star}, j^{\star}\right) \in F\left(i^{\star}, j^{\star}\right) .
\end{aligned}
$$

Hence $f$ is a selection of $F$ and $f\left(R_{1} \times R_{2}\right) \subset R_{1} \times R_{2}$. Moreover, by taking $\sigma_{1}=i d$ and

$$
\sigma_{2}= \begin{cases}i d, & \text { if } i^{*}<i^{\star} \text { and } j^{*}<j^{\star}, \text { or } i^{*}>i^{\star} \text { and } j^{*}>j^{\star} \\ \left(j^{*}, j^{\star}\right), & \text { otherwise, }\end{cases}
$$

we get (iii).

Case 2: When $\left(i^{*}, j^{*}\right)$ is not isolated, there exists $\left(i^{\star}, j^{\star}\right) \neq\left(i^{*}, j^{*}\right)$ such that $\left(i^{*}, j^{*}\right) \in F\left(i^{\star}, j^{\star}\right)$.

Case 2-1: When $i^{\star} \neq i^{*}$ and $j^{\star} \neq j^{*}$, by defining $R_{1}, R_{2}, f$ and $\sigma_{2}$ in the same way as Case 1 , we get (iii).

Case 2-2: When $i^{\star}=i^{*}$ and $j^{\star} \neq j^{*}$, by taking $R_{1}=\left\{i^{*}\right\}, R_{2}=\left\{j^{*}, j^{\star}\right\}$ and $f_{1}\left(j^{\star}\right)=i^{*}$, we get (i).

Case 2-3: When $i^{\star} \neq i^{*}$ and $j^{\star}=j^{*}$, we easily obtain (ii) as well as Case 2-2.

\section{An algorithm}

In this section, we present an algorithm for determining if a game is a partially monotone game.

\section{Algorithm. MAIN}

Input: Payoff matrices $A$ and $B$.

Output: TRUE or FALSE.

Step 0: Construct best response tables $R_{A}$ and $R_{B}$ as follows.

(0-1): For each $j=1,2, \ldots, n, R_{A}(j):=\left\{i^{\prime} \in S_{1}: a_{i^{\prime} j}=\max _{i \in S_{1}} a_{i j}\right\}$.

(0-2): For each $i=1,2, \ldots, m, R_{B}(i):=\left\{j^{\prime} \in S_{2}: b_{i j^{\prime}}=\max _{j \in S_{2}} b_{i j}\right\}$. 
Step 1: For each $T_{1} \subset S_{1}$ and $T_{2} \subset S_{2}$ with $\# T_{1}=\# T_{2}=2$, repeat the following.

(1-1): Check if $T_{1} \times T_{2}$ is a game or not by Algorithm CHECK-GAmE. Note that we describe procedure CHECK-GAME later. If it is true, then perform Step 1-2.

(1-2): Check if there exist a selection $f$ of $F$ and permutation $\sigma_{2}$ on $T_{2}$ such that the following hold:

$$
\begin{aligned}
& j<_{\sigma_{2}} j^{\prime} \Rightarrow f_{1}(j) \leqq_{i d} f_{1}\left(j^{\prime}\right) \text { for any } j, j^{\prime} \in T_{2}, \\
& i<_{i d} i^{\prime} \Rightarrow f_{2}(i) \leqq_{\sigma_{2}} f_{2}\left(i^{\prime}\right) \text { for any } i, i^{\prime} \in T_{1} .
\end{aligned}
$$

or not by Algorithm CHECK-Monotone. If it is true, then output TRUE and halt.

Step 2: For each $T_{1} \subset S_{1}$ and $T_{2} \subset S_{2}$ with $\# T_{1}=2$ and $\# T_{2}=1$, repeat the following.

(2-1): Check if $T_{1} \times T_{2}$ is a game or not by Algorithm Check-Game. If it is true, then output TRUE and halt.

Step 3: For each $T_{1} \subset S_{1}$ and $T_{2} \subset S_{2}$ with $\# T_{1}=1$ and $\# T_{2}=2$, repeat the following.

(3-1): Check if $T_{1} \times T_{2}$ is a game or not by Algorithm CHECK-Game. If it is true, then output TRUE and halt.

Step 4: Output FALSE.

\section{Algorithm. Check-Game}

Input: $T_{1}, T_{2}, R_{A}$ and $R_{B}$.

Output: TRUE or FALSE.

Step 1: If for any $j \in T_{2}, R_{A}(j) \cap T_{1} \neq \emptyset$ is satisfied, then go to Step 2. Otherwise output FALSE and halt.

Step 2: If for any $i \in T_{1}, R_{B}(i) \cap T_{2} \neq \emptyset$ is satisfied, then go to Step 3. Otherwise output FALSE and halt.

Step 3: Output TRUE.

Algorithm. Check-Monotone

Input: $T_{1}:=\left\{t_{11}, t_{12}\right\}\left(t_{11}<t_{12}\right), T_{2}:=\left\{t_{21}, t_{22}\right\}\left(t_{21}<t_{22}\right), R_{A}$ and $R_{B}$.

Output: TRUE or FALSE. to Step 2.

Step 1: (1-1) If $R_{A}\left(t_{21}\right)=\left\{t_{11}\right\}$ and $R_{A}\left(t_{22}\right)=\left\{t_{12}\right\}$, then let $\Sigma_{A}=\{i d\}$ and go Step 2.

(1-2) If $R_{A}\left(t_{21}\right)=\left\{t_{12}\right\}$ and $R_{A}\left(t_{22}\right)=\left\{t_{11}\right\}$, then let $\Sigma_{A}=\left\{\left(t_{21}, t_{22}\right)\right\}$ and go to

(1-3) Let $\Sigma_{A}=\left\{i d,\left(t_{21}, t_{22}\right)\right\}$

Step 2: (2-1) If $R_{B}\left(t_{11}\right)=\left\{t_{21}\right\}$ and $R_{A}\left(t_{12}\right)=\left\{t_{22}\right\}$, then let $\Sigma_{B}=\{i d\}$ and go to step 3. step 3 .

(2-2) If $R_{A}\left(t_{11}\right)=\left\{t_{22}\right\}$ and $R_{A}\left(t_{12}\right)=\left\{t_{21}\right\}$, then let $\Sigma_{B}=\left\{\left(t_{21}, t_{22}\right)\right\}$ and go to

(2-3) Let $\Sigma_{B}=\left\{i d,\left(t_{21}, t_{22}\right)\right\}$

Step 3: If $\Sigma_{A} \cap \Sigma_{B} \neq \emptyset$, output TRUE. Otherwise output FALSE.

THEOREM 4.1. This algorithm determines whether a two-person game is a partially monotone game. 
Proof. Thanks to Theorem 3.5, it suffices to check $2 \times 2-, 2 \times 1-$ and $1 \times 2-$ subgames to determine whether a two-person game is a partially monotone game. This yields our claim.

THEOREM 4.2. This algorithm requires $O\left(m^{2} n^{2}\right)$ time in total.

Proof. Since the numbers of combinations of $T_{1}$ and $T_{2}$ satisfying $\# T_{1}=\# T_{2}=$ 2 , $\# T_{1}=2$ and $\# T_{2}=1$, and $\# T_{1}=2$ and $\# T_{2}=1$ are

$$
\frac{m(m-1)}{2} \times \frac{n(n-1)}{2}, \frac{m(m-1)}{2} \times n \text { and } m \times \frac{n(n-1)}{2},
$$

respectively, Algorithm MAIN requires $O\left(m^{2} n^{2}\right)$ time. Further, Algorithms CHECKGame and CheCK-Monotone require constant time. Thus, this algorithm requires $O\left(m^{2} n^{2}\right)$ time in total.

\section{Concluding remarks}

At the beginning of this paper, we quoted an existence theorem of a pure-strategy Nash equilibrium based on monotonicity of the best responses. On the other hand, Iimura (2003) gave a class of games having a pure-strategy Nash equilibrium as an application of another type of discrete fixed point theorems from Iimura et al. (2005). Their theorem is based on Brouwer's fixed point theorem and relies on an integrally convex set. In Iimura (2003), he introduced direction preserving property for mappings, and presented the existence theorem by using it. However, this property is also not simple, so it is another interesting theme to create an algorithm for determining whether a game belongs to his class.

\section{Acknowledgement}

The authors thank Professor Hidefumi Kawasaki for his valuable suggestions and fruitful discussions for the first version of manuscript. In particular, Definition 3.1 and Lemma 3.2 were improved by his suggestions. We are also grateful to Professor Yoshihiro Mizoguchi for his useful support and comments.

In addition, this research was supported in part by a Grant-in-Aid for JSPS Fellows and Kyushu University Global COE Program "Education-and-Research Hub for Mathematics-for-Industry."

\section{References}

Iimura, T. (2003). A discrete fixed point theorem and its applications, J. Math. Econom., 39, 725-742.

Iimura, T., Murota, K. and Tamura, A. (2005). Discrete fixed point theorem reconsidered, J. Math. Econom., 41, 1030-1036.

Nash, J.F. (1950). Equilibrium points in n-person games, Proc. Nat. Acad. Sci. U.S.A., $36,48-49$.

Nash, J.F. (1951). Non-cooperative games, Ann. of Math. (2), 54, 286-295. 
Sato, J. and Kawasaki, H. (2009). Discrete fixed point theorems and their application to Nash equilibrium, Taiwanese J. Math., 13, 431-440.

Sato, J. and Kawasaki, H. (Preprint). Necessary and sufficient conditions for the existence of a pure-strategy Nash equilibrium.

Topkis, D. (1979). Equilibrium points in nonzero-sum $n$-person submodular games, SIAM J. Control Optim., 17, 773-787.

Received August 19, 2009

Revised October 6, 2009 